\title{
A MACHINE LEARNING APPROACH TO IMPROVING FORECASTING ACCURACY OF HOTEL DEMAND: A COMPARATIVE ANALYSIS OF NEURAL NETWORKS AND TRADITIONAL MODELS
}

\author{
MisukLee, Seattle University,leem@seattleu.edu \\ Xinpan Mu, Microsoft, xinpan.mu@outlook.com \\ Yiqiao Zhang, Expedia Group, sakuraisuri25@gmail.com
}

\begin{abstract}
Demand forecasting is a key component of successful hotel operations and revenue management. In this research, we suggest a combined neural network method that incorporates heterogeneous data sets - time series and advance booking information along with seasonality components. We provide a comparative analysis to improve forecasting accuracy by investigating various forecasting methods including advance booking models, time series models. Our exhaustive study shows that the neural network approach outperforms traditional forecasting models overall. Moreover, we observe that the true behavior of daily demand may be a more complex phenomenon than single type data can capture, and that our combined neural network model may improve forecasting accuracy.
\end{abstract}

Keywords: Forecasting, Time Series Neural Network, Combined Neural Network, Machine Learning, Hotel Demand.

\section{INTRODUCTION}

Accurate demand forecasting is a key component of successful hotel operations and revenue management due to the perishable nature of the products. While hotels have explored various forecasting methods, majority of those models fall into three main categories- advance booking models, time series models, and combined models (Lee 1990; Weatherford and Kimes 2003; Chen and Kachani 2007; Lee 2018). Advance bookings models use the patterns of reservation arrivals over a booking horizon to obtain the future demand to come. Time series models assume certain mathematical formula for historical patterns of the final demand. Combined models blend forecasts obtained from advance booking models and time-series models to increase the forecasting accuracy.

These traditional forecasting techniques, although they have shown overall satisfactory forecasting results in many tourism and hospitality applications, are often unable to capture complicated relationships of factors that may determine demand because they are restricted to certain mathematical functions. One technological advancement in the field of forecasting is neural networks. Neural networks are based on mathematical computing systems vaguely inspired by the biological neural networks that constitute brains (Hyndman and Athanasopoulos 2013). Neural network technology presents a highly flexible modeling capability to process complex relationships in large data sets. As neural networks are widely used for forecasting in many application areas including finance, engineering, and science, neural network models have been introduced as a new technique into the fields of tourism and hospitality demand forecasting including by Law 1998; Law and Au 1999; Law 2000; Cho 2003; Kon and Turner 2005; Pai and Hong 2005; Palmer et al. 2006; Chen 2011; Teixeira and Fernandes 2012; Claveria and Torra 2014; Claveria et al. 2015, and Sun et al. 2019.

While the above studies used neural network models to forecast tourist demand at the aggregate level (monthly or quarterly demand at the city or country level), there have been few published work applying neural networks (or machine learning techniques in general) to individual hotels, airlines, or more detailed levels of demand. Weatherford et al. 2003 applied neural network models to the weekly total number of reservations on a specific flight. The empirical results showed that a single layer neural network model may outperform traditional methods of moving average, exponential smoothing, and regression while the cubic regression model provided slightly better long-term forecasts. Antonio et al. 2017 developed several different machine learning models to predict hotel booking cancellations. Their comparative analysis using real booking data from four hotels demonstrated that machine learning techniques 
including neural networks and tree-based models can make it possible to predict booking cancellations with high accuracy.

These studies indicate a growing interest in using neural networks in the tourism and travel industry. Nevertheless, the majority of published articles is focused on high level demand forecasting. While neural network approaches have proven their capability in forecasting high level tourist demand, very few studies have applied neural networks to individual hotels, airlines, or more detailed levels of demand, partly due to high volatility and multi-echelon seasonality. In fact, high demand variability along with multi-seasonality often makes it challenging to predict daily hotel demand with high accuracy.

The purpose of this research is to develop neural network models for forecasting daily hotel demand. In order to properly capture the high demand volatility and multi-seasonality, we examine several different neural models involving time series data, as well as advance booking data, and propose combined neural network models to improve forecasting accuracy. Using real hotel data, we provide empirical results for the proposed model, along with a comparison to traditional forecasting methods. For proper validation, we conduct an exhaustive study of comparing the forecasting capability of neural networks to those of traditional methods. For the comparative analysis, we develop various time series models including ETS (simple exponential smoothing, Winter's, and Holt-Winter's) and ARIMA; advance booking models (additive model and multiplicative model); and traditional combined models.

The remainder of this paper is organized as follows. In section 2, we describe traditional forecasting methods such as advance booking models, ETS, ARIMA, and combined models. Then, we examine several different neural network models and propose a combined network model. Section 3 presents empirical results and comparative analysis. Finally, Section 4 includes conclusions and discussions.

\section{MODELS}

\section{Time Series Models}

Time series models derive forecasts based on the final number of rooms on a particular stay night. Time series models such as exponential smoothing in its various forms (ETS models) and ARIMA are traditional, yet still popular forecasting methods in the hospitality industry.

\section{Exponential Smoothing (ETS)}

Exponential smoothing methods has been used in travel industries for decades as simple, yet successful forecasting methods. Simple exponential smoothing, the first and simplest form of the ETS model, is suitable for forecasting data with no clear trend or seasonal pattern. Following notations of Hyndman and Athanasopoulos 2013, let $y_{t}$ denote the final demand for time (stay date) $t$ and $\hat{y}_{t}$ the forecast for stay date $t$. When a sequence of observations begins at time $t=0$, the simple exponential smoothing is formulated as follows:

$$
\hat{y}_{t+1}=\alpha y_{t}+(1-\alpha) \hat{y}_{t}, \quad \hat{y}_{0}=y_{0}
$$

where $\alpha$ is the data smoothing factor, $0<\alpha<1$. That is, $\hat{y}_{t+1}$, the forecast for the next period $t+1$, is the weighted average of a new observation $y_{t}$ and the previous forecast $\hat{y}_{t}$.

Double exponential smoothing or Holt's method extended simple exponential smoothing to allow forecasting of data with a trend. This method involves a forecast equation and two smoothing equations (one for the level and one for the trend). Triple exponential smoothing or Holt-Winters' seasonal method extend simple exponential smoothing method to capture trend and seasonality. With trend component $b_{t}$ and seasonal component $s_{t}$, this method consists of the forecast equation and three smoothing equations of level, trend, and seasonality (Hyndman and Athanasopoulos 2013). There are different types of seasonality: multiplicative and additive. The $h$-step-ahead forecast $\left(\widehat{y}_{t+h}\right)$ of Holt-Winters' smoothing with additive seasonality is given by

$$
\begin{array}{ll}
\hat{y}_{t+h}=l_{t}+b_{t}+s_{t+h^{-} m} & \text { (Forecas) } \\
l_{t} & =\alpha\left(y_{t}-s_{t-m}\right)+(1-\alpha)\left(l_{t-1}+b_{t-1}\right)
\end{array}
$$




$$
\begin{aligned}
& b_{t}=\beta\left(l_{t}-l_{t-1}\right)+(1-\beta) b_{t-1} \quad \text { (Trend) } \\
& \begin{array}{lll}
s_{t} & =\gamma\left(y_{t}-l_{t-1}-b_{t-1}\right)+(1-\gamma) s_{t-m} & \text { (Seasonality) }
\end{array}
\end{aligned}
$$

where $\beta$ is the trend smoothing factor, $0<\beta<1, m$ the number of seasons in a given period (year, month, week, etc.) and $\gamma$ the smoothing factor for seasonality.

Similarly, the $h$-step-ahead forecast $\left(\widehat{y}_{t+h}\right)$ of Holt-Winters' smoothing with multiplicative seasonality is given by

$$
\begin{aligned}
\hat{y}_{t+h} & =\left(l_{t}+b_{t}\right) s_{t+h^{-m}} & & \text { (Forecast) } \\
l_{t} & =\alpha\left(y_{t} / s_{t-m}\right)+(1-\alpha)\left(l_{t-1}+b_{t-1}\right) & & \text { (Level) } \\
b_{t} & =\beta\left(l_{t}-l_{t-1}\right)+(1-\beta) b_{t-1} & & \text { (Trend) } \\
s_{t} & =\gamma\left(y_{t} /\left(l_{t-1}-b_{t-1}\right)\right)+(1-\gamma) s_{t-m} & & \text { (Seasonality) }
\end{aligned}
$$

\section{Auto Regressive Integrated Moving Average (ARIMA)}

According to Hyndman and Athanasopoulos (2013), "While exponential smoothing models are based on descriptions of level, trends, and seasonality in the data, ARIMA models describe auto-correlations in the data". More specifically, ARIMA models consist of two components - autoregression and moving average. Autoregression models forecast the variable of interest using a linear combination of past values of the variable. "The term autoregression indicates a regression of the variable against itself. Moving average models use past forecast errors in a regression-like model" (Hyndman and Athanasopoulos 2013).

If we denote $B$ the backward shift, i.e. $B y_{t}=y_{t-1}$, ARIMA models combine differencing with autoregression and a moving average model. The non-seasonal ARIMA model can be written as

$$
\begin{array}{rrr}
\left(1-\varphi_{1} B-\ldots-\varphi_{p} B^{p}\right)(1-B)^{d} y_{t}= & c+\left(1+\theta_{1} B+\ldots+\theta_{q} B^{q}\right) \varepsilon t \\
A R(p) & \text { differences } & M A(q)
\end{array}
$$

where $(1-B)^{d} y_{t}$ is the differenced series, $\left(1-\varphi_{1} B-\ldots-\varphi_{p} B^{p}\right)$ the autoregression term, and $\left(1+\theta_{l} B+\ldots+\theta_{q} B^{q}\right)$ the moving average term. This is also called $\operatorname{ARIMA}(p, d, q)$, where $p$ is the order of the autoregression (AR) component, $d$ the degree of differencing, and $q$ the order of the moving average (MA) component. ARIMA models can also extend to accommodate seasonal data. A seasonal ARIMA model is formed by including additional seasonal terms in a nonseasonal ARIMA model. One shorthand notation for the model is $\operatorname{ARIMA}(p, d, q)(P, D, Q)_{s}$, with $D$ the seasonal differencing, $Q=$ seasonal MA order, and $S$ the time span of the repeating seasonal pattern.

\section{Advance Booking Models}

Advance bookings models use arrival patterns of reservation requests over a booking horizon. At any point on a booking horizon, on-the-book number (current bookings on hand) reflects partial demand. By adding this early realization to the estimated number of future bookings to come, we can forecast the final demand.

While advance booking models are widely used in the hotel business, majority of advance booking approaches can be categorized into two types - additive models and multiplicative models (Lee 2018). Additive models are based on the assumption that the bookings on hand at any given lead time is independent of future bookings to come (Lee 2018). Additive models estimate remaining bookings using the historical booking profiles, and then the final demand is computed as the sum of on-the-book number and estimated number of future bookings. Multiplicative models assume that the number of bookings to come is proportional to the current number of bookings on hand (Lee 2018). Thus, the final demand is derived as the product of the historical average booking rate (ratio of on-the-book to final demand) and the current number of bookings. In this research, we implement both additive and multiplicative pick-up models.

\section{Combined Models}

The basic idea of combined models is to blend forecasts obtained from different forecasting methods to obtain the final forecasts. While there is no optimal mathematical solution for the weights and coefficients when mixing different models, combined models are generally adopted in practice, and different heuristic methods including that of Rajopadhye et al. 2001 are evaluated to obtain better forecasts. Generalizing combined models, we develop regression 
models of time series forecasts and advance booking forecasts. For example, we can obtain a regression combined model by fitting daily demand to ARIMA forecasts and additive pick-up forecasts.

\section{Neural Network Models}

Among several architectures of neural network models, the most widely used model in forecasting is the backpropagation multilayer perceptron (MLP) model. As Figure 1 illustrates, a MLP neural network consists of three or more layers: an input layer, an output layer, and at least one hidden layer. The output of a node (neuron) is computed as

$$
O_{i}=g\left(\sum w_{i j} I_{j}\right) .
$$

where $O_{i}$ is the output of node $i, g$ is the activation function, $w_{i j}$ the weight from node $i$ to node $j$, and $I_{j}$ the input from node $j$.

Commonly used activation functions include sigmoid (logistic), piece-wise linear (Rectified Linear Unit), and step function, as presented below.

$$
\begin{array}{ll}
g(x)=\frac{1}{1+e^{-x}} & \text { sigmoid(logistic) } \\
g(x)=\max (0, x) & \text { piecewise linear } \\
g(x)= \begin{cases}1 \quad \text { if } x \geq 0 \\
0 & \text { if } x<0\end{cases} & \text { step function }
\end{array}
$$

The hidden layers, along with the non-linear activation function, make neural networks learn and model non-linear and complex relationships between inputs and outputs. Moreover, many studies have shown that neural network framework can provide better models of data with high volatility and heteroscedasticity, as neural networks are capable of learning unknown complex relationships in the data without imposing any fixed mathematical restrictions on the data. This property is very useful in time series forecasting, in which data volatility is high. Previous research in neural network modeling for tourism demand, including Palmer et al. (2006) and Chen (2011), also adopted the time series approach.

As shown in Figure 2, seasonality and high demand volatility are most critical issues in hotel demand forecasting. Especially, high variability of demand is apparent in more detailed (disaggregated) levels of demand, such as daily demand. As our first neural network model, we develop a time series neural network model that feeds historical daily demand into input nodes.

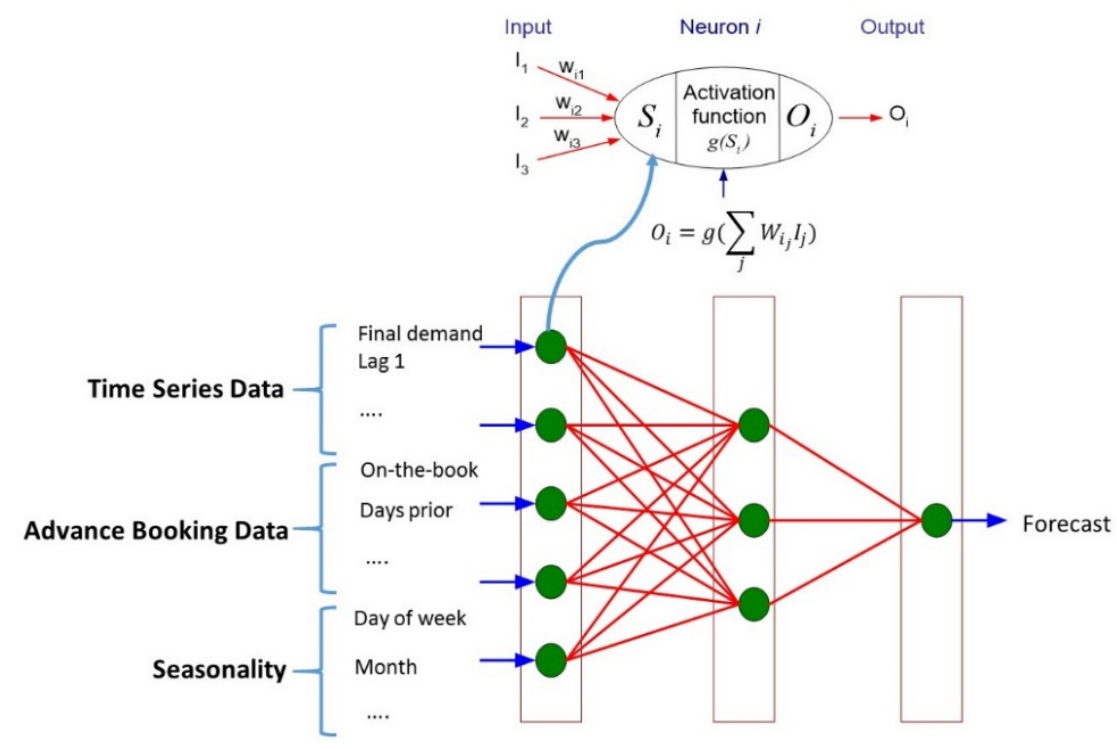

Figure 1. Combined Neural Network Model 
While time series data may be a good predictor for trend and seasonality, advance booking information may be a very accurate prognosticator of final demand for a particular stay night in short-term forecasting. Thus, we propose an advance booking neural network model. In the traditional advance booking models, booking curves concern only current on-the-book numbers and ignore the previous booking profiles. However, recent research on advance booking models such as Tse and Poon (2015), and Lee (2018) points out that considering trends and patterns in the booking pace may help better model recent seasonality or demand shifts. Thus, to learn booking patterns and trends, our advance booking neural network models use not only bookings-on-hand but also booking pace for each stay date.

Table 1. Variables for Combined Neural Network Model

\begin{tabular}{|c|c|c|}
\hline \multicolumn{2}{|r|}{ Variable } & Description \\
\hline \multirow{4}{*}{$\begin{array}{c}\text { Booking } \\
\text { Data }\end{array}$} & days prior & Number of days prior to stay date \\
\hline & on-the-book & Cumulative bookings for given days prior \\
\hline & otb-lag1, otb-lag7, otb-lag14 & Lagged values for cumulative bookings \\
\hline & avg remaining bookings & Historical average remaining demand for given days prior \\
\hline $\begin{array}{l}\text { Time } \\
\text { Series }\end{array}$ & $\begin{array}{l}\text { lag1,.., lag7, } \\
\text { lag14, lag21, lag28, lag91, } \\
\text { lag182, lag363, lag364, } \\
\text { lag365 }\end{array}$ & Lagged values for daily final demand \\
\hline \multirow{3}{*}{$\begin{array}{l}\text { Seasonality } \\
\text { Factor }\end{array}$} & day of week & Day of week dummy variables. Monday,...,Sunday \\
\hline & Month & Month dummy variable \\
\hline & avg DOW demand & Historical average final demand by day of week. \\
\hline
\end{tabular}

Time series data may capture historical trend and seasonality, and advance booking information may reflect most recent demand shifts. However, the true behavior of future demand may be a more complex phenomenon. In fact, one key advantage of neural network models is the ability to model non-linear and complex relationships. Thus, we suggest a combined neural network model, which incorporates time series data and advance booking information. Moreover, as neural networks do not impose restrictions on input variables, we include seasonality components that traditional time series models such as ARIMA and ETS models are not capable of handling. For example, exploratory data analysis shows that both demand level and variability are significantly different by day of week (for intended stay date) in a specific month or season. Also, booking patterns are significantly different by day of week and remaining days prior to stay date. We feed those hotel-specific seasonal and time variables into the combined neural network model presented in Table 1.

In this research, we tested 14 different forecast methods as presented in Table 2. Note that while developing the time series models, we perform experiments to optimize model parameters using training data. For ETS models, we use the smoothing factor $(\alpha)$, trend factor $(\beta)$, and seasonality factor $(\gamma)$ that minimize training errors. We tune the ARIMA parameters $p, q, d, P, D$, and $Q$ in Equation (3) to find the best ARIMA models. Also, for each type of neural network model, we try different combinations of activation functions (step function, logistic, and piece-wise linear) and numbers of layers along with numbers of nodes, to find the best models. 
Table 2. List of Models Developed

\begin{tabular}{|c|c|c|}
\hline \multicolumn{2}{|c|}{ Type } & Model \\
\hline \multirow[t]{5}{*}{ Time Series } & \multirow[t]{3}{*}{ ETS } & Simple Exponential Smoothing \\
\hline & & Holt's Trend Model \\
\hline & & Holt-Winters' Model \\
\hline & \multirow[t]{2}{*}{ ARIMA } & Non-seasonal ARIMA \\
\hline & & seasonal ARIMA \\
\hline \multirow{2}{*}{\multicolumn{2}{|c|}{ Advance Booking }} & Additive Pick-up \\
\hline & & Multiplicative Pick-up \\
\hline \multirow{4}{*}{\multicolumn{2}{|c|}{ Regression Combined }} & Best ARIMA + Additive Pick-up (No Intercept) \\
\hline & & Best ARIMA + Additive Pick-up + Intercept \\
\hline & & Best ARIMA + Additive Pick-up + Day of Week (No Intercept) \\
\hline & & Best ARIMA + Additive Pick-up + Day of Week + Intercept \\
\hline \multirow{3}{*}{\multicolumn{2}{|c|}{ Neural Network }} & Time Series Neural Network \\
\hline & & Advance Booking Neural Network \\
\hline & & Combined Neural Network \\
\hline
\end{tabular}

\section{EMPIRICAL RESULTS}

The data set is comprised of daily bookings at two hotels from a major hotel chain and collected for the period 1 May 2008 to 30 April 2010. The last six months of data is reserved for model validations. Figure 2 shows the daily final demand used in this paper. Several interesting facts emerge from the figure. First, high variance in demand is apparent at both hotels. While high demand variation is a common phenomenon in many fields of the tourism and travel industry, these two hotels seem to experience quite high demand volatility. More importantly, we note that daily hotel-level demand is much more volatile than aggregate-level demand, which makes daily hotel forecasting more challenging. Second, the seasonality of these two hotels is relatively less obvious or quite irregular except for the day of week seasonality. In general, seasonality is considered a key factor that determines the level of room demand. Irregular seasonality along with high variance in demand may hinder accurate forecasting of room demand. This motivated us to develop advanced forecasting models that are capable of non-linear multi-echelon complexities of seasonality and demand variation.

We compare the performance of proposed neural network models with traditional approaches in terms of predictive accuracy for daily hotel demand. For a benchmark, we use a naive model, which we define as the demand 364 days before the day we are forecasting (same day, last year). This is one of the most popular benchmark models in the hotel business as it considers multiple aspects of seasonality (day of week, weekly, monthly) at the same time and it often shows quite a good prediction accuracy. 

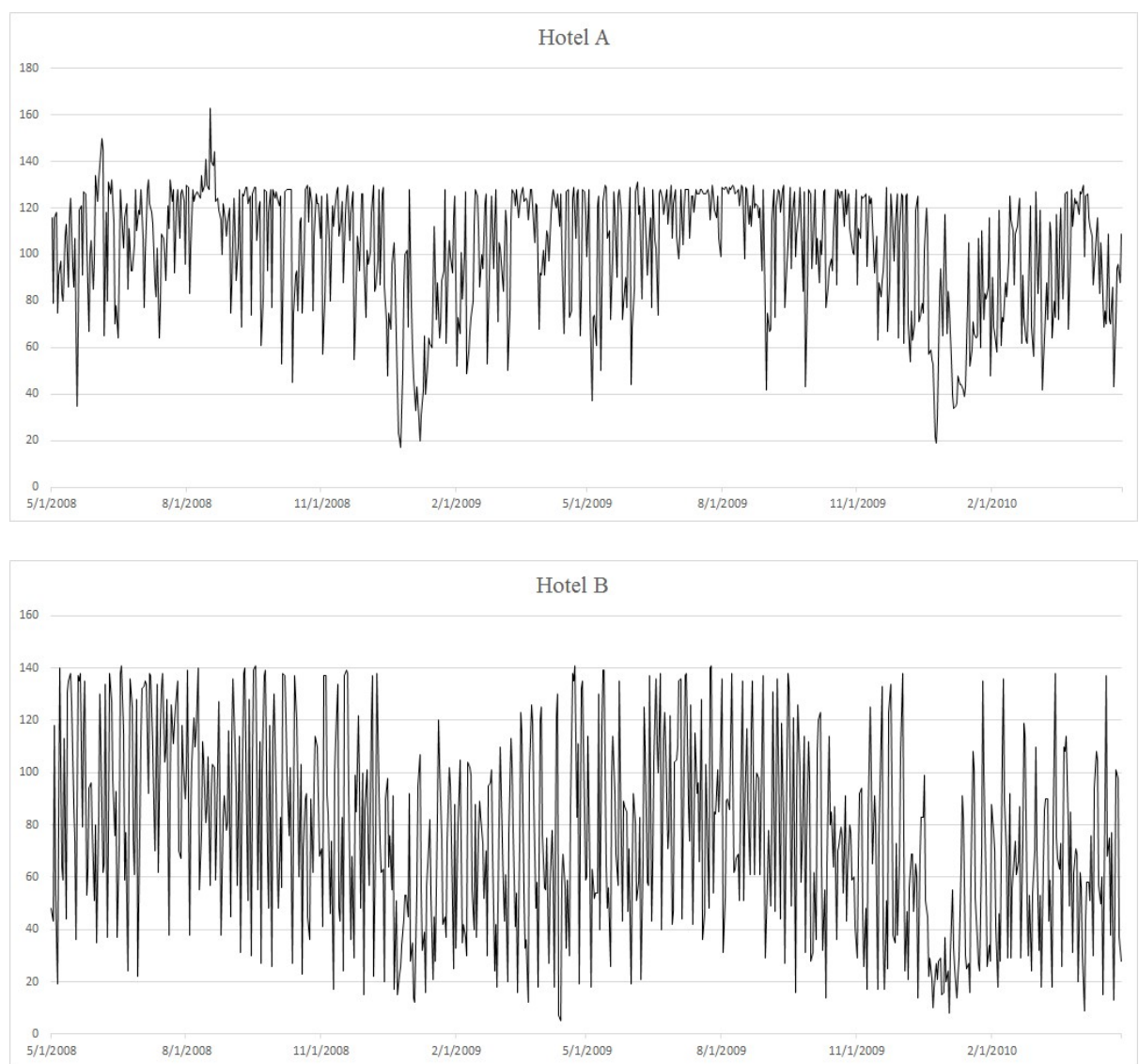

Figure 2: Daily Room Demand (Hotel A and Hotel B)

In order to evaluate the forecasting performance of different models, we use two error measures - MASE (Mean Absolute Scaled Error) and GMRAE (Geometric Mean Relative Absolute Error). These two scale-independent measurements involve dividing the forecast error by the error obtained using a naive model.

$$
\begin{gathered}
\text { MAPE }=\sum_{t} \frac{\left|A_{t}-F_{t}\right|}{\left|A_{t}-F_{t}{ }^{\prime}\right|} \\
\text { GMRAE }=\operatorname{geomean}\left(r_{t}\right)=\sqrt[k]{\prod_{t=1}^{k} \frac{\left|A_{t}-F_{t}\right|}{\left|A_{t}-F_{t}{ }^{\prime}\right|}}
\end{gathered}
$$

where $A_{t}$ denotes the actual value, $F_{t}$ the forecast value of the suggested model, and $F_{t}$ ' the naive forecast value. Note that both measures require a naive model. MASE is a generally applicable measurement of forecast accuracy even when the data exhibit a trend or a seasonal pattern (Hyndman 2006). An alternative approach to compute scale-free error measures is to use individual relative absolute error $r_{t}$. The geometric mean (GMRAE) to average the absolute relative errors is generally more desirable than either the arithmetic mean (MRAE) or the median (MdRAE) since it tends to produce more robust estimates on relative errors (Davydenko and Fildes 2013). 
Table 3: Forecasting Results

\begin{tabular}{|c|c|c|c|c|c|c|c|c|}
\hline \multirow[b]{2}{*}{ Hotel } & \multirow[b]{2}{*}{ Model } & \multirow[b]{2}{*}{ Selected Model } & \multicolumn{3}{|c|}{ MASE } & \multicolumn{3}{|c|}{ RMRAE } \\
\hline & & & 1 month & $\begin{array}{c}3 \text { months } \\
\text { out }\end{array}$ & $\begin{array}{c}6 \text { months } \\
\text { out }\end{array}$ & $\begin{array}{c}1 \text { month } \\
\text { out }\end{array}$ & $\begin{array}{c}3 \text { months } \\
\text { out }\end{array}$ & $\begin{array}{c}6 \text { months } \\
\text { out }\end{array}$ \\
\hline \multirow[t]{7}{*}{ A } & Advance Booking & Additive & 0.83 & 1.09 & 1.21 & 0.86 & 1.13 & 1.32 \\
\hline & ETS & Simple Exponential & 1.17 & 1.44 & 1.51 & 1.35 & 1.61 & 1.56 \\
\hline & ARIMA & $(1,1,1)(3,1,1)_{7}$ & 1.02 & 1.48 & 1.40 & 1.04 & 1.58 & 1.45 \\
\hline & Combined & ARIMA + Additive & 0.86 & 1.16 & 1.44 & 0.90 & 1.29 & 1.44 \\
\hline & \multirow{3}{*}{ Neural Network } & NN Advance Booking & 0.74 & 0.94 & 1.34 & 0.71 & 0.96 & 1.34 \\
\hline & & NN Time Series & 0.92 & 0.90 & $\underline{1.04}$ & 0.96 & $\underline{0.89}$ & $\underline{1.01}$ \\
\hline & & NN Combined & $\underline{0.68}$ & $\underline{0.89}$ & 1.10 & $\underline{0.71}$ & 1.00 & 1.16 \\
\hline \multirow[t]{7}{*}{ B } & Advance Booking & Additive & 0.91 & 1.07 & 1.11 & 0.95 & 1.08 & 1.15 \\
\hline & ETS & Holt Winters & 0.91 & 1.24 & 0.93 & 0.91 & 1.27 & 0.91 \\
\hline & ARIMA & $(2,1,1)(3,1,1)_{7}$ & 0.91 & 1.24 & 0.90 & 0.86 & 1.26 & 0.91 \\
\hline & Combined & ARIMA + Additive & 0.76 & 1.06 & 1.00 & 0.85 & 1.13 & 0.93 \\
\hline & \multirow{3}{*}{ Neural Network } & NN Advance Booking & 0.69 & 0.87 & 1.08 & 0.73 & 0.88 & 1.14 \\
\hline & & NN Time Series & 0.88 & 1.11 & 0.87 & 0.93 & 1.04 & $\underline{0.88}$ \\
\hline & & NN Combined & $\underline{0.65}$ & $\underline{0.76}$ & $\underline{0.84}$ & $\underline{0.71}$ & $\underline{0.78}$ & 0.94 \\
\hline
\end{tabular}

We applied the 14 forecasting methods to the data of the two hotels. We consider a booking horizon of six months (i.e. days prior $=0,1, \ldots, 180$ days). Table 3 shows the average MASE and GMRAE values for three neural network models along with the best models from each of the four traditional forecasting methods (ETS, ARIMA, Advance Booking, and Combined Model).

Recall that MASE represents the ratio of average forecasting errors of the given model to those of the naive model. For example, one month out MASE of the combined neural network model for Hotel A in Table 3 is 0.68 , which means that the total forecast error of the combined neural network is $68 \%$ of that of the naive model. GMRAE provides similar interpretations. For example, one month out GMRAE of Hotel A reports a geometric average ratio of the combined neural network errors to those of the naive model of 0.71 .

Based on the results in Table 3, we obtain the following insights. First, neural network approach outperform the traditional models for both hotels and across all forecasting horizons. The time series neural network and advance booking neural network achieve forecasting accuracy superior to that of the traditional advance booking model and the best time series model, respectively. This implies that actual demand behavior may be more complicated than simple booking curves or specific functional form of time series can capture, and that neural networks may provide more flexible modeling capability in handling many complexities of daily demand patterns and/or seasonality differences.

Second, within the neural network models, time series models tend to show better performance in the long-term forecasting (6 months out), and advance booking models tend to achieve higher accuracy in short to medium term forecasting (1-3 months out). This result is consistent with common practice in the hotel business and in previous studies including Rajopadhye et al. 2001 and Lee 2018. As the intended stay date comes closer, more booking requests arrive, and advance booking information reflects the final demand more accurately. Hence, advance booking models tend to produce lower errors in short-term forecasting.

Finally, the combined neural network is overall superior to the traditional models and other neural network models, whereas the time series neural network models may produce slightly better results in certain cases of long-term forecasting. We note that combined neural network models may significantly lower forecasting errors compared to the case of using advance booking or time series data alone. Especially, the fact that combined models achieves better short-term (one month out) forecasting results may suggest that we can better model actual demand patterns by incorporating advance booking information with time series data. 
Moreover, the combined neural network model may be more robust than other models. Both error measures of the combined neural network model are relatively consistent across all lead times. For example, MASE of Hotel B is 0.65 for 1 month out, 0.76 for 3 months out, and 0.84 for 6 months out, whereas MASE values of other models significantly vary with days prior.

\section{CONCLUSION}

Accurate forecasting of room demand is a key component of hotel operations and management. In this paper, we performed exhaustive research on various forecasting methods for daily hotel room demand. We developed 14 different models across three categories of forecasting approaches - advance booking models, time series models, and neural network models. The forecasting results obtained from actual hotel data demonstrate that the neural network approach outperforms the traditional forecasting models including advanced time series models. This implies that neural network models may provide more flexible modeling capability to capture non-linear and complex relationships hidden in daily hotel demand.

Most of all, this research suggests a neural network method that incorporates time series data with advance booking information along with other seasonality components. The true behavior of daily demand may be a more complex phenomenon than a single type of data can capture. The features of complex demand patterns intertwined across different time periods and over different reservation horizons have been less intensively studied owing to the modeling complexity required. However, the presence of different data sources for demand patterns can be leveraged for accurate forecasting. Our empirical study has shown that combined neural network models may improve the shortterm to medium-term forecasting accuracy.

In this research, we have demonstrated that back-propagation MLP neural network models may increase forecasting accuracy. Moreover, we investigated different types of activation functions and different numbers of layers and nodes to find the best MLP models. Future research may include a study using different neural network architectures such as recurrent neural networks (Elman network, Jordan network, long short-term memory network, etc.) and recursive neural networks (tensor network) to see if any, further improvement is possible.

\section{REFERENCES}

Antonio, N., De Almeida, A. \& Nunes, L. (2017). Predicting hotel booking cancellation to decrease uncertainty and increase revenue. Tourism and Management Studies, 13, 25-39.

Chen, C. \& Kachani, S. (2007). Forecasting and optimisation for hotel revenue management. Journal of Revenue and Pricing Management, 6(3), 163-174.

Chen, K.-Y. (2011). Combining linear and nonlinear model in forecasting tourism demand. Expert Systems with Applications, 38(8), $10368-10376$.

Cho, V. (2003). A comparison of three different approaches to tourist arrival forecasting. Tourism Management, 24(3), $323-330$.

Claveria, O., Monte, E., \& Torra, S. (2015). Tourism demand forecasting with neural network models: Different ways of treating information. International Journal of Tourism Research, 17(5), 492-500.

Claveria, O. \& Torra, S. (2014). Forecasting tourism demand to Catalonia: Neural networks vs. time series models. Economic Modelling, 36, 220 - 228.

Davydenko, A. \& Fildes, R. (2013). Measuring forecasting accuracy: The case of judgmental adjustments to sku-level demand forecasts. Journal of Forecasting, 29, 510-522.

Hyndman, R. J. (2006). Another Look at Forecast Accuracy Metrics for Intermittent Demand. Foresight: The International Journal of Applied Forecasting, 1(4), 43-46.

Hyndman, R. J. \& Athanasopoulos, G. (2013). Forecasting: principles and practice, 1st Edition. OTexts.

Kon, S. C. \& Turner, L. W. (2005). Neural network forecasting of tourism demand. Tourism Economics, 11(3), 301328.

Law, R. (1998). Room occupancy rate forecasting: a neural network approach. International Journal of Contemporary Hospitality Management, 10(6), 234-239.

Law, R. (2000). Back-propagation learning in improving the accuracy of neural network-based tourism demand forecasting. Tourism Management, 21(4), 331 -340. 


\section{Issues in Information Systems}

Volume 21, Issue 1, pp. 12-21, 2020

Law, R. \& Au, N. (1999). A neural network model to forecast Japanese demand for travel to Hong Kong. Tourism Management, 20(1), 89 - 97.

Lee, A. (1990). Airline reservations forecasting: Probabilistic and statistical models of the booking process. Ph.D. thesis.

Lee, M. (2018). Modeling and forecasting hotel room demand based on advance booking information. Tourism Management, 66, $62-71$.

Pai, P.-F. \& Hong, W.-C. (2005). An improved neural network model in forecasting arrivals. Annals of Tourism Research, 32(4), $1138-1141$.

Palmer, A., Montao, J. J., \& Ses, A., 2006. Designing an artificial neural network for forecasting tourism time series. Tourism Management, 27(5), 781 - 790.

Rajopadhye, M., Ghalia, M. B., Wang, P. P., Baker, T., \& Eister, C. V. (2001). Forecasting uncertain hotel room demand. Information Sciences, 132(14), $1-11$.

Sun, S., Wei, Y., Tsui, K.-L., \& Wang, S. (2019). Forecasting tourist arrivals with machine learning and internet search index. Tourism Management, 70, 1- 10.

Teixeira, J. P., \& Fernandes, P. O. (2012). Tourism time series forecast -different ANN architectures with time index input. Procedia Technology 5, 4th Conference of ENTERprise Information Systems aligning technology, organizations and people (CENTERIS 2012), $445-454$.

Tse, T. S. M., \& Poon, Y. T. (2015). Analyzing the use of an advance booking curve in forecasting hotel reservations. Journal of Travel \& Tourism Marketing, 32(7), 852-869.

Weatherford, L. R., Gentry, T. W., \& Wilamowski, B. (2003). Neural network forecasting for airlines: A comparative analysis. Journal of Revenue and Pricing Management, 1(4), 319-331.

Weatherford, L. R. \& Kimes, S. E. (2003). A comparison of forecasting methods for hotel revenue management. International Journal of Forecasting, 19(3), 401-415. 